\title{
Review of: "Spatiotemporal change analysis of long time series inland water in Sri Lanka based on remote sensing cloud computing"
}

Zhiqiang Tan

Potential competing interests: The author(s) declared that no potential competing interests exist.

Compared with the traditional process of data retrieval -> download -> preprocessing -> spatial analysis -> mosaicking and clipping -> mapping, GEE provides a more efficient solution for the storage and statistical analysis of big earth data. Using the massive remote sensing data and powerful statistical capabilities provided by GEE to study the spatial-temporal dynamics of surface water bodies has achieved a series of pioneering results, and it will undoubtedly become an important trend in hydrology research.

In general, this study on the extraction of lakes, reservoirs, and rivers based on the water index and automatic threshold technology is not the first. The "innovation" acquired from "the research on the spatiotemporal variation of inland water in Sri Lanka is still in the blank" limits the broad attention of this study.

Even though water is an easily identified ground cover type, the overlap accuracy of $99.14 \%$ is very high. However, given that the comparison is "based on the visual interpretation of the water boundary" and 100 test samples might be selected artificially, the accuracy is not entirely credible. Especially for mudflats with high moisture content, shallow water covered by vegetation canopy, and water bodies with high sediment content, even visual interpretation cannot guarantee sufficient accuracy. 